\title{
Correction
}

\section{Cross-Tachyphylaxis of the Pressor Response to Angiotensins in Conscious Rabbits}

\author{
Brian P. Rowe, Alan R. Noble, and Kenneth A. Munday \\ Department of Physiology and Pharmacology, The University, Medical and Biological Sciences Building, \\ Bassett Crescent East, Southampton, S09 3TU. Great Britain
}

Pflügers Arch. 380, 13-17 (1979)

On page 15 , lines $1-3$ should read:

Ang I $\left(1.0-10 \mu \mathrm{g} \cdot \mathrm{kg}^{-1}\right)$, Ang II $\left(0.1-1.0 \mu \mathrm{g} \cdot \mathrm{kg}^{-1}\right)$, Ang III $\left(1.0-10 \mu \mathrm{g} \cdot \mathrm{kg}^{-1}\right)$ and NA $(3.0-$ $\left.30 \mu \mathrm{g} \cdot \mathrm{kg}^{-1}\right)$.

On page 15, Fig. 2. Ang II doses shown should read:

$0.1 \mu \mathrm{g} / \mathrm{kg}, 0.3 \mu \mathrm{g} / \mathrm{kg}, 1.0 \mu \mathrm{g} / \mathrm{kg}, 170 \mu \mathrm{g} / \mathrm{kg}, 0.1 \mu \mathrm{g} / \mathrm{kg}, 0.3 \mu \mathrm{g} / \mathrm{kg}, 1.0 \mu \mathrm{g} / \mathrm{kg}, 0.1 \mu \mathrm{g} / \mathrm{kg}$.

On page 15, Fig. 3, Horizontal axis should read:

$\%$ change in blood pressure.

Also legend to Fig. 3 should start:

Mean percentage reduction in pressor response... 